W73

IS INFECTION AN IYPORTANT DETERYINANT OF THE OUTCOYE OF PANCREATIC NECROSIS ? D.Alexander, M. Madan, P. Curley.

A.Chalmers, R. Fomler, M.J.Makahon. University Department of Surgery, The General Infirmary at Leeds, Great George Street, Leeds LSI 3EX.

Infection is believed to be important to the development of the 'septic' syndrone which complicates severe pancreatitis, and an indication for operative intervention. In order to evaluate the role of infection in the pathogenesis of severe acute pancreatitis a prospective study was carried out in 23 patients with pancreatic necrosis who underwent percutaneous aspiration at the time of either computed tomography (CT) or ultrasound examination. Patients were initially treated conservatively, (including 'pig-tail'drainage of the pancreatic collection) and operative treatment using debridement and external drainage was used if the patient's condition deteriorated.

Twelve patients were infected and 11 sterile. Four patients with sterile necrosis died, three after surgery had been performed, whilst there was one death (post-operative) in the group with infected necrosis. There was no significant difference in the APACHE II scores between the 2 groups (median value of 5.5 for the infected group $v 6$ for the sterile group).

These results suggest that the presence of infection in an area of pancreatic necrosis cannot be determined clinically, and that infection is not an important determinant of outcome if percutaneous drainage is used in it's management. Infection was not an indication for operative intervention.
Biliary tract W75-W82

W75
THE NATURAL HISTORY OF GALL STONES IN DIABETES: A FIVE YEARS FOLLOW-UP

G. Del Favero, F. Di Mario, A. Caroli, A. Volpi, A. Puglisi P. Scalon, T. Meggiato, R. Naccarato

Dep. of Gestroenterology, University of Padua, ITALY

Few data are available in the literature on the natural history of gall stones in diabetics, subjects who present a high prevalence of the disease In these subjects cholecystectory is often performed even if they are asymptomatic.

This study was undertaken to assess the natural history of gall stones in patients with diabetes and associated cholelithiasis.

81 patients with diabetes and associated gall stones were studied. In all subjects diagnosis was made by ultrasound at the time of presentation. After 5 years from the diagnosis of gall stones the patients were called and requested to answer an anamestic questionnaire. They were also submitted to physical examination and, whether with galldladder in situ, to a second ultrasound. Subjects were divided in to two graps: group 1: patients asymptomatic at the first diagnosis; grap 2: symptomatic (previaus episode ( $\mathbf{s}$ ) of biliary pain).

3 out of 81 patients could not be found; 8 had died from diseases not related to gallstones. Therefore, we could evaluate 70 subjects (50 female 20 male, mean age $63.8 \pm$ SD 8.7 yrs). 47 out of these belonged to grap 1 , 23 to grap 2. The amulative percentage of patients who presented biliary pain and were submitted to cholecystectomy during the follow-up was $14.8 \%$ and $17 \%$ in grap $1,47.6 \%$ and $21.7 \%$ in grap 2, respectively. 1 subject (1.4\%) died after cholecystectomy. The presence of gall stones was confirmed in 34/35 (97.1\%) patients who underwent the ultrasonographic examination.

Natural history of gall stones in diabetics does not seem to differ from that reported for general population: while asymptomatic patients become symptomatic in a low percentage of cases (which do not seem to differ from that described in not diabetics), about a half of initially symptomatic subjects presented episodes of pain in the following five years and ane fourth were operated. On the basis of these data cholecystectory is not justified in diabetics with asymptomatic gall stones but a conservative approach is more advisable.
IS MONOCYTE FUNCTION IMPAIRED IN COMPLICATED PANCREATITIS ?

D.Alexander, Madan, P.Curley,S.Switala,

J.Hodason. R.King. M. Mclyahon.

University Department of Surgery, The

General Infirmary at Leeds, Great George street, Leeds LSI 3EX.

Activated protease, released into the plasma from the pancreas during acute pancreatitis, are complexed by $\alpha_{2}^{-}$ macroglobulin. The complexes retain potentially damaging peptidase activity but are usually eliminated promptly by mononuclear phagocytes. In severe acute pancreatitis about 308 of plasma $\alpha_{2}^{-}$ macroglobulin is complexed suggesting impaired clearance. The aim of this study was to examine the phagocytic index of monocytes, isolated from the plasma of patients with acute pancreatitis, by assessing their incorporation of methyl $14 \mathrm{C}$-trypsin. The severity of the acute illness was assessed by the acute physiology score (APS) and the results compared to control

volunteers from the Department of Surgery. Fourteen patients with complicated acute pancreatitis (protracted attack, abscess or necrosis) were studied on 29 occasions_yith a median phagocytic index (PI) of $6.4 \times 10^{-6}$

This value was not significantly different when compared to 6 control volunteers studied on 14 occasions ( $P I=5 \times 10^{-6}$ ). Six patients were in a critical state at the time of study (5 deaths) with a significantly higher median APS (11 v 4) compared to the remaining 8 patients (no deaths). The PI was higher in the patients with systenic manifestations of severe disease $\left(7.4 \times 10^{-6}\right.$ v $\left.5.3 \times 10^{-6}\right)$ although not significantly so.

We have found no evidence of diminishment of the phagocytic capacity of monocytes in complicated acute pancreatitis.
TNF INHIBITOR EXISTS IN BLOOD OF PATIENTS WITH OBSTRUCTIVE JAUNDICE

MCA PUNTIS WG JIANG

University Department of Surgery, University of wales College of Medicine, Cardiff.

Tumour necrosis factor inhibitor (binding protein) has been reported recently in patients with cancer or pyrexia. We studied the inhibitor in blood from jaundiced patients who are known to have raised monocyte TNF levels. 27 patients with obstructive jaundice were studied. Plasma was separated before clinical intervention. TNF inhibitor was assayed using an 1929 cell TNF cytotoxic inhibition assay as described recently (1). The results are shown as percentage inhibition, by plasma, on the cytotoxicity of TNF $(40 \mathrm{U} / \mathrm{ml})$ on 1929 cells. Jaundiced patients showed increased inhibition $7.0 \pm 1.48$ compared with controls $(2.5 \pm 1.28$, $p<0.05$ ). There is no difference between benign jaundice $(5.3 \pm 3.18)$ and malignant $(7.7 \pm 1.5 \%$, $p=0.3)$. These levels are not related to bilirubin level $(r=0.18, p>0.05)$, bile salt $(r=-$ 0.32 , p<0.05) nor TNF production by monocytes $(r=0.16, \quad P>0.05)$. However 5 patients with malignant obstruction or advanced tumours, who died shortly after the test had significantly lower levels of TNF inhibitors $(3.2 \pm 1.82$ vs $9.3 \pm 1.8 \%$ than the other patients, $p<0.05$ ) suggesting that patients with advanced disease lack the inhibitor to protect them from TNF induced damage as shown in mild sepsis or trauma.

We conclude that patients with obstructive jaundice possess an inhibitor in their blood especially in those with malignant obstructions. The lower level of this inhibitor may relevent to patient prognosis.

(1). Baughman et al. An inhibitor of tumor necrosis factor found in pleural effusions. Lab Clin Med. 1991;118:326 


\section{A PROSPECTIVE STUDY COMPARING BRUSH AND BILE EXFOLIATIVE CYTOLOGY FOR DIAGNOSING BILE DUCT STRICTURES.}

T Kurzawinski, B Davidson, J Dooley, R Dick, A Deery and K Hobbs. Hepatobiliary and Liver Transplantation Unit and Department of Cytology, Royal Free Hospital and School of Medicine, Pond Street, London NW3 2QG

Imaging of biliary strictures may suggest malignancy but cytology can provide a tissue diagnosis. The aim of this study was to compare the diagnostic value of brush (BRC) and bile (BIC) cytology.

Thirty two patients ( 20 males, 12 females, median age 66 years, range 31-84) with biliary strictures at ERCP (24) or PTC (8) had both BIC and BRC. Brushings were taken using a modified Geenan cytology brush ( $6 \mathrm{Fr}$, Wilson Cook) passed alongside a guide wire which remained through the stricture. Bile was aspirated after insertion of an internal/external catheter or an endoprosthesis. Bile was examined by one experienced cytologist $(\mathrm{AD})$ and was reported as positive or negative for malignant cells.

Twenty-nine patients had malignant strictures. 16 were confirmed by histology and 13 had malignancy suggested by clinical follow up. Three patients had histologically benign strictures. The overall sensitivity of BRC (17 of $29+\mathrm{ve}, 59 \%$ ) was significantly greater than BIC $(7$ of $29+v e, 24 \%)(p<0.01)$ as was the diagnostic accuracy ( 63 vs $31 \%, p<0.01$ ). The specificity for both was $100 \%$ and the sensitivity by cancer type was:

$\begin{array}{lccc} & \text { Pancreas } & \text { Bile duct } & \text { Am pulla } \\ \text { Bile } & 31 \% & 11 \% & 0 \% \\ \text { Brush } & 50 \% & 56 \% & 100 \%\end{array}$

None of the patients had positive bile cytology with negative brush cytology. There were no procedure related complications and the average sampling time was $<5$ minutes.

We conclude that brush cytology is more sensitive than bile cytology and with the technique described is safe and rapid.
W79

\section{PATTERNS OF GALLSTONE MANAGEMENT IN A GEALTH DISTRICT} A P BARLOH, I A EYRE-BROOK

Taunton \& Somerset MBS Trust, Musgrove Park, Taunton

The management of gallstone disease in a health district of population 250,000 was studied prospectively in three separate 12 month periods: (i) $1987 / 88$ before endoscopic biliary therapy (ET) or laparoscopic cholecystectomy (LC) were available (ii) $1989 / 90$ when ET available (iii) 1990/91 when ET and LC available. Patien numbers increased [period (i) $n=181$; (ii) $n=205$; (iii) $n=264$ ] reflecting: a) more patients $>75$ years treated for duct stones (DS) [(i) $n=9 ;$ (ii) $n=24 ;$ (iii) $n=28$ ] b) more cholecystectomies as vaiting list eroded [(i) $n=181$; (ii) $\mathrm{n}=191$; (iii) $\mathrm{n}=224]$ c) 38 population increase. DS were increasingly managed with ET alone [(ii) 16 of $63=25 \%$; (iii) 38 of $58=66 \%$ ] with no mortality and low morbidity [pancreatitis $n=1$; haemorrhage $n=1$; perforated gastric ulcer $n=1$ ]. In contrast 4 of 100 died after surgery for DS. of 54 ET patients 42 (77\%) had their gallbladder in situ and none subsequently required cholecystectony. In periods (ii) and (iii) 23 of 39 (598) with pancreatitis and DS received $\mathrm{ET}$ alone. The retained stone rate following surgery for DS fell with use of intraoperative choledochoscopy (IC) [(i) 5 of 20 ; (iii) 0 of 16]. During period (iii) one surgeon performed $98 \mathrm{LC} ; 16$ required ERCP and 5 LC were converted to laparotomy. Theatre time was longer for IC [141+51 mins] than open cholecystectomy $(O C)$ [ $91 \pm 26$ mins] but hospital stay was shorter [LC $n=2$ days OC $n=6$ days]. 90 were back at full activity within 14 days of LC with only 1 episode of minor umbilical wound sepsis

We conclude that ET, IC and LC have all improved the management of gallstone disease. ET provided sufficient and safe therapy for DS in most elderly patients. If LC + ET is accepted as treatment for DS in younger patients the need for ERCP will be greater than that predicted by the BSG working party. In period (iii) ve performed 77 ERCP procedures for gallstone disease and we calculate a need for 99 ERCP per annum if all DS are managed endoscopically.
DECRRASED KUPTFSR CEST CHEARAMCE CAPACITY (RCCC)

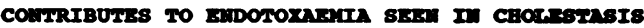
B Clements, I Halliday, $P$ Irwin, $X$ Mccaique and B J Rowlands (introduced by MF $R$ J roorehead) Dept.of Surgery, Queen's University, Belfast BT12 6BJ. w.Ireland.

A novel single pass hepatic perfusion technique using an FITC-labelled latex probe was developed for measuring RCCC and applied in an animal model of biliary obstruction. Six groups $(n=12)$ of male wistar rats $(250-$ 350g) were perfused - Control 1 (no operation), Control 2 (sham operation), BDI (Bile duct ligated) animals for 1 , 2, 3 and 4 weeks. Plasma bilirubin, endotoxin and anticore glycolipid concentrations were assayed. $\mathrm{xCCC}$ was calculated and expressed as a percentage. Rats jaundiced for greater than two weeks had a significantly decreased $\mathrm{xCCC}(p<0.001)$. Significantly higher endotoxin concentrations and anticore glycolipid concentrations correlated positively with hyperbilirubinaenis ( $p<0.001)$. Anti-core glycolipid concentrations correlated strongly with systemic endotoxaemia $(p<0.00001)$ and both were inversely correlated with duration of $\mathrm{BDL}(\mathrm{p}<0.01)$.

\begin{tabular}{|l|l|l|l|l|l|l|}
\hline MODEL & Ho op. & shan & BDL 1 & BDI.2 & BDL.3 & BDL4 \\
\hline Rcce (8) & 34.4 & 38.7 & 41 & 27.2 & 15.4 & 6.2 \\
& \pm 3.82 & \pm 3.74 & \pm 5.03 & \pm 5.43 & \pm 5.22 & \pm 2.28 \\
\hline Endotoxin & 8.3 & 9.3 & 30.3 & 31.7 & 33.8 & 55.1 \\
pg/al & \pm 4.5 & \pm 6.3 & \pm 17.4 & \pm 21 & \pm 16.5 & \pm 40.1 \\
\hline Anti-core & 102.4 & 111.7 & 179.3 & 283 & 319.1 & 326.9 \\
glycolipid & \pm 7.6 & \pm 37.8 & \pm 123.2 & \pm 162.6 & \pm 139.8 & \pm 147.4 \\
\hline Bilirubin & 1.6 & 0.9 & 192.7 & 162.9 & 167.8 & 152.1 \\
ol/1 & \pm 0.71 & \pm 0.64 & \pm 21 & \pm 25 & \pm 20 & \pm 23 \\
\hline
\end{tabular}
Conclusion : Maximal hyperbilirubinaemia precedes reduced RCCC. Anti-core glycolipid concentration accurately reflect chronic endotoxin exposure. Impairment of kupffer. cell function may be responsible for the endotoxaemia associated with cholestasis.
ABSENCE OF POST-OPERATIVE IMMUNOSUPPRESSION AFTER LAPAROSCOPIC SURGERY.

$J$. Griffith, $H$, Sue-Ling, $N$, Everitt, $A$. Boylston, D. Johnston, M.J. McMahon. Academic Unit of Surgery \& Department of Pathology, The General Infirmary, Leeds.

Laparoscopic surgery offers several advantages over conventional surgery in the treatment of gallstones: less surgical trauma and more rapid recovery after operation. Hence, we wondered whether the "normal" postoperative immunosuppression might be less after laparoscopic surgery than after conventional operative methods.

To test this hypothesis, T-cell

proliferation was measured before and after operation in 18 patients who underwent laparoscopic cholecystectomy and 8 patients who underwent conventional cholecystectomy. Mixed populations of T-lymphocytes were stimulated in vitro with phytohaemaglutinin (PHA)

staphylococcal enterotoxin B (SEB) and toxic shock syndrome toxin (TSST) and proliferation assessed by uptake of tritiated thymidine.

Results were expressed as a stimulation index = stimulated activity - basal activity RESULTS

Surgery basal activity

Laparoscopi

PHA $175(53-234)$
SEB $101(31-146)$
TSST $84(27-120)$

Post-op $03(58-190)$ NS $98(37-138)$ NS TSST 84( 27-120) 62(31-108)NS

Conventional PHA 169(106-206) 34( 8- 85)* SEB $129(69-154) \quad 53(11-61)$ * TSST 118(67-136) 29(14-48)* Medians and interquartile ranges; ${ }^{*} p<0.0$

Significant immunosuppression was seen after conventional surgery but not after laparoscopic surgery. These findings may have implications for the surgical treatment of high risk patients for other conditions. 
W81

COMBINED PERCUTANEOUS AND ENDOSCOPIC BILIARY STENTI:IG: SUCCESS AND COMPLICATIONS.

M.B. Sheridan, D.E.F. Tweedle, D.F. Martin.

Departments of Radiology and Surgery, Gastro-intestinal Un1t, Withington Hospital, University Hospital of South Manchester, M20 8LR

Rellef of biliary obstruction by endoscopic stent insertion is successful in the majority of cases, with a complication rate of about $10 \%$. In patients in whom endoscopic stenting falls, combined percutaneous and endoscopic procedures are useful although a high complication rate has been reported. We have reviewed our results in 45 consecutive patients over a recent 18 month period. A stazed procedure under antibiotic cover and with careful attention to fluid balance was used after the first failed E.R.C.P.

External drainage was established in all cases, and a successful combined procedure performed in 43 $(96 \%)$. Of the 2 unsuccessful cases, 1 patient died prior to completion of the combined procedure anc 1 patient required surgical drainage.

Complications occured in $33 \%$ of patients with $\equiv 30$ day mortality of $4 \%$. Mild cholangitis was the commonest complication occuring in 10 (22\%). Other complications included haemobilia in 3 , subphrenic collection in 1 and severe right upper quadrant sain in 2. All complications settled on conservaiive treatment requiring no other active intervention.

Cur complication rate for initial ERCP,

Percutaneous drainage and combined procedure together is higher than for endoscopic stenting alone, but all complications were minor and treated conservatively. Combined procedure is successful in most patients and complications can be kept to a minimum by rigorous use of antibiotic and fluid regimens with early recourse to staged combined procedure when initial E.R.C.P. falls.

PALLIA'IVE LAPAROSCOL'LC CHOLFCYSTENTEROS'WIMY FOR

OBSTRUCTIVE JAUNDICE IN ADVANCED CANCER OF THE HEAD OF THE PANCREAS.

S.W. Banting, S.M. Shimi, A. Cuschieri.

Department of Surgery, Ninewells Hospital \& Medical School Dundee, DDl 9SY.

Palliative relief of obstructive jaundice is indicated in advanced cancer of the head of the pancreas. This has been achieved by surgical bypass or by the insertion of an endoprosthesis.

We report on laparoscopic cholecystenterostomy as a new method of biliary drainage in advanced pancreatic cancer. four patients (IM 3F) mean age 73 years $(63-80)$ with obstructive jaundice due to cancer of the head of the pancreas were found to have metastatic disease at staging laparoscopy. Laparoscopic cholecystjejunostomy was performed at the same procedure after operative cholangiography had demonstrated a safe distance between the cystic-CBD junction and the obstructing lesion. The: operating time was $150-225$ minutes. Rapid relief of jaundice without complications was achieved in three patients. In the fourth jaundice persisted after initial. improvement. On re-intervention, the anastomosis was found to be obstructed by debris. Suction aspiration of this was followed by rapid relief of jaundice. Median post-operative hospital stay was 12 days $(5-36)$. In the follow-up period of 2-7 months all patients remained free of jaundice and no paient required readmission.

This technique provides an alternative method of palliative relief of obstructive jaundice in pancreatic cancer.

\section{W83}

Motility

W83-W92
EFFECT OF SODIUM RHEIN ON THE MOTILITY OF THE VASCULARLY PERFUSED RAT LARGE BOWEL IN VITRO. P.E.Squires. R.D.E.Rumsey N.W.Read. Dept of Biomedical Science, University of Sheffield, S10 2TN, UK

The aim of the present study is to show whether the Senna glycoside sodium Rhein, excites or modifies large bowel motility. 12 male Wistar rats were anaesthetised using sodium pentabarbitone (Sagatal, $60 \mathrm{mg} / \mathrm{kg}$, I.P.). The large bowel, including $10 \mathrm{~cm}$ of terminal ileum, was isolated and perfused vascularly with oxygenated Krebs bicarbonate buffer $\left(95 \% \mathrm{O}_{2} / 5 \% \mathrm{CO}_{2} ; 37^{\circ} \mathrm{C}\right.$ at $1.2 \mathrm{ml} / \mathrm{min}$ ) via the superior mesenteric artery, with hepatic portal outflow. The in-vitro preparation was suspended horizontally between two volume transducers, in a Krebs filled organ bath. Serosal strain gauges recorded contractility in the tail (CT) and body (CB) of the caecum, the proximal (PC), proximal-mid (PM), middistal (MD) and distal colon (DC). Aboral fluid flow was measured by volume transducer. Krebs solution ( $\mathrm{pH}=7.4$ ) infused luminally for 30 min, produced a profile of basal activity, before either a $2^{\text {nd }}$ Krebs infusion, or Krebs containing sodium Rhein ( $1 \mathrm{mM}, \mathbf{n}=6$ $\mathrm{pH}=7.4$; Madaus, Cologne). A final 30 min Krebs wash followed. Each period is subdivided into $10 \mathrm{~min}$ intervals, with data expressed as mean \% change in activity \pm sem, from basal levels, recorded as area under the response from each gauge site/transducer. Data is also obtained on propulsive activity.

Activity preceeding the administration of Rhein is similiar to control, except in the $2^{\text {nd }} 10 \mathrm{~min}, \mathrm{MD}$ and FF $(89 \% \pm 9,110 \% \pm$ 9 respectively; $P<0.05$ ). In the 10 mins immediately after administration, contractility significantly increases in CT $(159 \% \pm$ 42), CB $(153 \% \pm 28), P C(117 \% \pm 9), P M(128 \% \pm 26)$ and DC $(121 \% \pm 15 ; \mathrm{P}<0.05$ in each case). This effect is transient in the colon, being negated by the $2^{\text {nd }} 10$ min interval. In the caecum, the effect is sustained throughout administration, CT, $2^{\text {nd }} 10 \mathrm{~min}$ $(160 \% \pm 25)$ and $C B, 2^{\text {nd }}$ and $3^{\text {rd }} 10 \min (202 \% \pm 42,180 \% \pm$ 33 repectively; $\mathrm{P}<0.05)$. The number of propagated complexes significantly increases significantly by the $2^{\text {nd }}$ and $3^{\text {rd }} 10 \mathrm{~min}$ interval of the challenge $(3.7 \pm 0.7,3.7 \pm 0.4$ respectively; $P<$ 0.05 ), a response reflected in the increase in fluid flow throughout administration $(143 \%+14,154 \% \pm 27$ and $154 \% \pm 18$ respectively; $P<0.05,0.05$ and 0.01 ). The data suggests that $\mathbb{R}_{\text {hein }}$ acts to excite all motility transiently in the colon, before shifting the pattern of motility to that of sustained propulsion.
AMBULATORY MOTILITY PATTERNS OF THE TRANSPOSED SHORT SEGMENT COLON.

G.Peppas, H.R.Payne, J.Federico, K.Jeyasingham.

With the advent of ambulatory manometry fhere is currently some documentation of the motility of the left hemi-colon. We have performed ambulatory manometry on 10 patients who had undergone lower oesophageal replacement with the left hemi-colon over a $2-20$ year period. A simultaneous recording of the ambient $\mathrm{pH}$ in the intrathoracic colon was also carried out. In every patient the recording included upright, supine, prandial and postprandial periods. The results were analysed both visually as well as with the Gastrosoft Multigram Software, Version 5 (Synectics). The emerging motility patterns showed 3 basic types of waves based on amplitudes: Low Amplitude Contractions (LAC) measuring < $60 \mathrm{mmHg}$, Medium Amplitude Contractions (MAC) measuring 60-100mmH, and High Amplitude Contractions (HAC) at $100-200 \mathrm{mmHg}$. Further subtypes were ident if led. The LAC's showed Sporadic (amp. $40 \mathrm{mmHg}$, dur.7sec), Repetitive (same amp. / dur., but lasting 5 mins.) and Bursting (amp. $25 \mathrm{mmHg}$, dur.5sec. and lasting 5-20 mins.) waves. The MAC' two 5 subtyes of wave forms: secs.) and a bell-shaped one (dur. 13 secs.). The HAC's were seen in 3 patients with a duration of 15 secs. and were seen in 3 patients with a duration of 15 secs.' and patients however pat lents however, a plateau wave form with an amplitude of $20 \mathrm{mmHg}$ and lasting $20-30$ secs. Occurred post-prandially $(14.00-15.00 \mathrm{hrs})$, features resembling the gastro-colic reflex. Using the software which was primarily devised for oesophageal motility, we were able to distinguish propulsive (68-558) from simultaneous and/or mixed waves. The higher proportion of propulsive waves were noted in patients who had been operated on ten or more years previously. We may therefore conclude that the transposed colon retains its normal motility pattern, 